\title{
Spondylodiscitis with Epidural and Psoas Muscle Abscesses as Complications After Transrectal Ultrasound-guided Prostate Biopsy: Report of a Rare Case
}

\author{
Chiao-Ching Li ${ }^{1,2}$, Chiao-Zhu Li ${ }^{1,3}$, Sheng-Tang Wu'2, Tai-Lung Cha², Shou-Hung Tang ${ }^{2}$ \\ ${ }^{1}$ Department of Surgery, Kaohsiung Armed Forces General Hospital, Kaohsiung, Taiwan \\ ${ }^{2}$ Division of Urology, Department of Surgery, Tri-Service General Hospital, National Defense Medical Center, Taipei, Taiwan \\ ${ }^{3}$ Department of Neurological Surgery, Tri-Service General Hospital, National Defense Medical Center, Taipei, Taiwan
}

\begin{abstract}
Received: 05/07/2017
Accepted: 08/07/2017
\end{abstract}

Published: 02/08/2017

How to cite this article: Li CC, Li CZ, Wu ST, Chat TL, Tang SH. Spondylodiscitis with epidural and psoas muscle abscesses as complications after transrectal ultrasound-guided prostate biopsy: report of a rare case. EJCRIM 2017;4: doi:10.12890/2017_000694.

Conflicts of Interests: The Authors declare that there are no competing interests.

Acknowledgements: We would like to thank Editage (www.editage.com) for English language editing and publication support.

This article is licensed under a Commons Attribution Non-Commercial 4.0 License

\section{ABSTRACT}

A 71-year-old man presented with spondylodiscitis with epidural and psoas muscle abscesses following transrectal ultrasound (TRUS)guided prostate biopsy. These rare complications were detected by computed tomography of the abdomen and magnetic resonance imaging of the lumbar spine. The patient was successfully treated with antibiotics and underwent neurosurgery. Awareness of presentations such as backache and weakness of the lower limbs after prostate biopsy is important as these symptoms are usually mistaken for bone or muscle problems and often not recognized as being related to infection.

\section{LEARNING POINTS}

- We describe the case of a patient who experienced two major complications (spondylodiscitis with epidural abscess and psoas muscle abscess) following prostate biopsy.

- Awareness of these potential complications following prostate biopsy is essential to prevent life-threatening consequences.

\section{KEYWORDS}

Spondylodiscitis; prostate biopsy; transrectal ultrasound; psoas muscle abscess

\section{INTRODUCTION}

Approximately 1 million transrectal ultrasound (TRUS)-guided prostate biopsies are performed annually in the United States. The procedure is considered safe, but minor complications are common and include haematuria, rectal haemorrhage and haematospermia. Major complications, such as infections, are occasionally observed.

Here, we report the case of a patient who experienced two major complications (spondylodiscitis with epidural abscess and psoas muscle abscess) following prostate biopsy. He presented with fever, backache and lower limb weakness. We describe this case in order to highlight these complications as most physicians are not aware these problems can occur after prostate biopsy. 


\section{CASE REPORT}

A 71-year-old Taiwanese man presented with nocturia and incomplete voiding, which had occurred intermittently for 2 years. A digital rectal examination (DRE) revealed an enlarged prostate but no hard nodule. Urinalysis was negative for pyuria and haematuria. The prostatespecific antigen level was $7.845 \mathrm{ng} / \mathrm{ml}$, and the TRUS-measured prostate volume was $64 \mathrm{ml}$. A biopsy was performed before a 5-alpha reductase inhibitor ( $5 \alpha \mathrm{RI})$ was administered. Preparation for the procedure included mandatory cessation of anticoagulants, cleansing of the rectum and antibiotic consumption. Levofloxacin $750 \mathrm{mg}$ was prescribed for oral use 1 day before the biopsy.

TRUS revealed homogenous echogenicity and no hypoechoic lesions. Twelve core prostatic tissue samples were collected. Gross haematuria was observed after the biopsy, so a Foley catheter was placed for drainage. The patient was observed for 24 hours until no fever was evident. The Foley catheter was removed with a normal voided volume of $150 \mathrm{ml}$. The patient was discharged and resumed normal activities.

However, 1 day after discharge the patient noted a fever of $38.5^{\circ} \mathrm{C}$, which was accompanied by backache and severe myalgia in the right thigh. His vital signs were as follows: temperature, $38.5^{\circ} \mathrm{C}$; heart rate, 103 beats/minute; respiratory rate, 18 counts/minute; and blood pressure, 107/63 mmHg. DRE revealed a tender prostate. Leucocytosis (white blood cell count: 9550/ $\mathrm{\mu l}$ ) was not observed, but neutrophil predominance (neutrophil: 94\%), elevated C-reactive protein $(23.36 \mathrm{mg} / \mathrm{dl})$, elevated procalcitonin $(23.3 \mathrm{ng} / \mathrm{ml}$ ) and pyuria (white blood cells: >100/high power field) were noted.

Initially, prostatitis with sepsis was considered, and $2 \mathrm{~g}$ of once-daily intravenous ceftriaxone was started. However, the backache still persisted after 3 days of treatment with ceftriaxone. The patient's condition worsened and he developed shortness of breath, so he was transferred to the intensive care unit for care.

Plain radiography of the lumbar spine and computed tomography (CT) of the abdomen were performed. Radiographic images revealed narrowing of the L3-L5 vertebrae (Fig. 1), while a cystic lesion about $3.3 \mathrm{~cm}$ in size over the right psoas muscle was observed on CT (Fig. 2). The cystic lesion could not be drained via a percutaneous approach. We continued antibiotic treatment with ceftriaxone for 2 days and scheduled the patient for magnetic resonance imaging (MRI) of the lumbar spine for backache evaluation, which revealed abscesses in the $L 3 / 4$ and $L 4 / 5$ intervertebral disc spaces, along with narrow enhancement of the L3-L5 vertebral bodies and abnormally enhanced lesions involving both psoas muscles (Fig. 3 A and B).

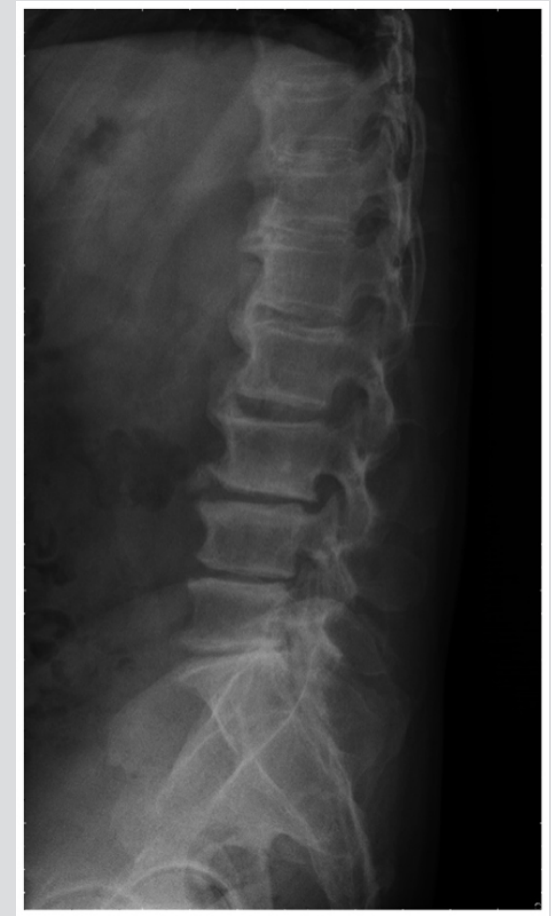

Figure 1. Radiograph of the lumbar spine (sagittal view) on the 2nd day of hospitalization shows narrowing of the L3-L5 vertebrae

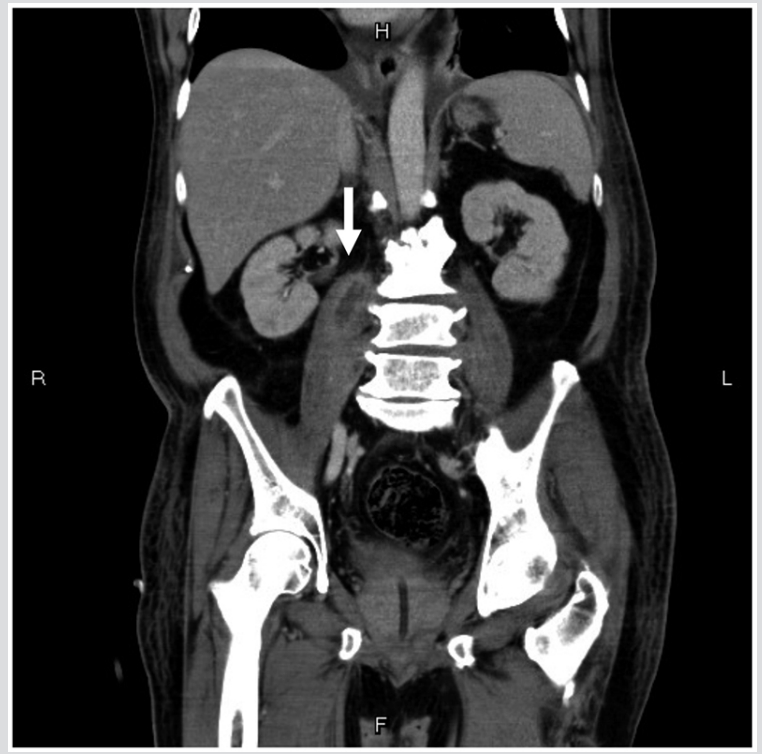

Figure 2. A computed tomography image of the abdomen (coronal view) during the 2 nd week of hospitalization shows a cystic lesion approximately $3.3 \mathrm{~cm}$ in size over the right psoas muscle with wall enhancement (white arrowhead), which could not be drained via the percutaneous approach 

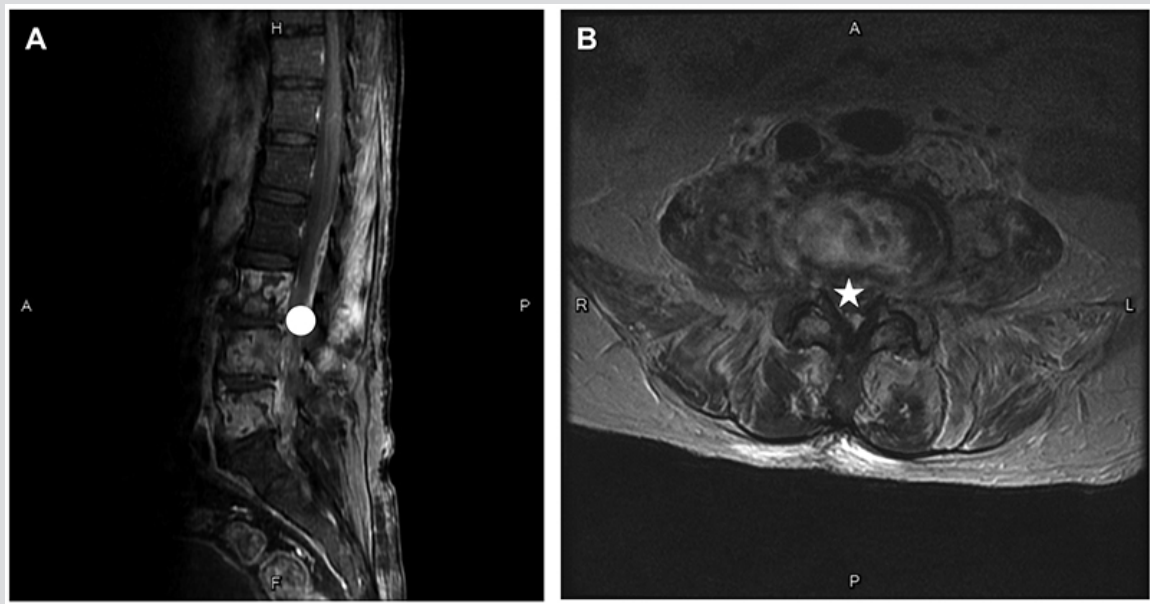

Figure 3. Images taken on the patient's 21st day of hospitalization. (A) Magnetic resonance image of the lumbar spine (sagittal view) shows fluid collection in the L3/4 and L4/5 intervertebral disc spaces together with narrow enhancement of the L3-L5 vertebral bodies (circle). (B) Magnetic resonance image of the lumbar spine (axial view) shows abnormally enhanced lesions involving the bilateral psoas muscles, prevertebral space and intraspinal canal (star)

A spiking fever (up to $39^{\circ} \mathrm{C}$ ) with chills developed. Escherichia coli identified in a blood culture within 3 days, showed multiple drug resistance in a urine culture (resistant: cefazolin, ceftriaxone, ciprofloxacin and levofloxacin; susceptible: flomoxef, piperacillin-tazobactam, cefepime, doripenem and imipenem). Subsequently, after 5 days of use, ceftriaxone was switched to $0.25 \mathrm{~g}$ of intravenous doripenem three times daily. Neurosurgeons performed total laminectomies of the L4/L5 vertebrae and removal of the epidural abscesses. Pathology revealed chronic inflammation of the spine and epidural tissue. The patient was discharged on the seventh postoperative day and returned to normal activities.

\section{DISCUSSION}

TRUS-guided prostate biopsy is a universal procedure for diagnosing prostate cancer and is recommended before $5 \mathrm{aRI}$ is administered. Spondylodiscitis with epidural abscess and psoas muscle abscess after prostate biopsy are extremely rare complications ${ }^{[1]}$.

The annual incidence of infectious spondylodiscitis ranges from 0.4 to 2.4 per 100,000 cases. Haematogenous dissemination of E. Coli from the genitourinary tract is the most common cause. The internal vertebral venous plexus is considered the main pathway for spreading metastatic prostate cancer by providing continuity with the prostatic venous plexus. Based on Batson's hypothesis ${ }^{[2]}$, prostate tumor cells reach the vertebral venous plexus of the spine, particularly under transient conditions of increased intra-abdominal pressure, and form metastatic tumour deposits in the vertebrae. This valveless plexiform venous network could also provide a potential route for the spread of infection.

The psoas muscle's blood supply is derived from the four lumbar arteries, and venous drainage occurs via lumbar veins. The pathogen disseminates by this route to form psoas muscle abscesses.

The optimal solution for spondylodiscitis is surgery, which is also indicated for epidural abscesses and septicaemia resistant to targeted antibiotic therapy. The first-line treatment for a psoas muscle abscess is a broad-spectrum antibiotic. The safe alternative is percutaneous drainage, and the last approach to consider is open surgery ${ }^{[3]}$.

Predisposing factors for abscesses include older age, immunosuppression and diabetes mellitus. Moreover, rectal enema should be considered as a standard bowel preparation in patients undergoing biopsy ${ }^{[4]}$.

Infection after prostate biopsy is increasing because of the development of resistance to fluoroquinolone ${ }^{[5]}$. It is important to be aware of the potential complications described above. In this case, we successfully treated the patient with removal of epidural abscesses after diagnosing spondylodiscitis and psoas muscle abscesses based on findings from CT and MRI of the lumbar spine. Physicians should be aware of the possibility of these complications when patients present with backache and lower limb weakness after prostate biopsy so that lifethreatening progression can be avoided.

\section{REFERENCES}

1. Dobson G, Cowie CJ, Holliman D. Epidural abscess with associated spondylodiscitis following prostatic biopsy. Ann R Coll Surg Engl 2015 ;97:e81-e82.

2. Nathoo N, Caris EC, Wiener JA, Mendel E. History of the vertebral venous plexus and the significant contributions of Breschet and Batson. Neurosurgery 2011;69:1007-1014.

3. Shields D, Robinson P, Crowley TP. Iliopsoas abscess-a review and update on the literature. Int J Surg 2012;10:466-469.

4. De Nunzio C, Lombardo R, Presicce F, Bellangino M, Finazzi Agro E, Gambrosier MB, et al. Transrectal-ultrasound prostatic biopsy preparation: rectal enema vs. mechanical bowel preparation. Cent European J Urol 2015;68:223-228.

5. Rudzinski JK, Kawakami J. Incidence of infectious complications following transrectal ultrasound-guided prostate biopsy in Calgary, Alberta, Canada: a retrospective population-based analysis. Can Urol Assoc J 2014;8:E301-E305. 